\title{
$A b$ initio density functional theory study of the electronic, dynamic, and thermoelectric properties of the crystalline pseudobinary chalcogenide $(\mathrm{GeTe})_{x} /\left(\mathrm{Sb}_{2} \mathrm{Te}_{3}\right)(x=1,2,3)$
}

\author{
Wilfredo Ibarra-Hernández ${ }^{1,2, *}$ and Jean-Yves Raty ${ }^{2,3}$ \\ ${ }^{1}$ Facultad de Ingeniería-BUAP, Apartado Postal J-39, Puebla, Pue. 72570, México \\ ${ }^{2}$ Départment de Physique, Université de Liège, Allée du 6 Août 17, B-4000 Sart Tilman, Belgium \\ ${ }^{3}$ UGA, CEA, LETI, MINATEC campus, 17 rue des Martyrs, F-38054 Grenoble Cedex 9, France
}

(Received 17 November 2017; revised manuscript received 23 April 2018; published 15 June 2018)

\begin{abstract}
We use $a b$ initio density functional theory calculations to understand the electronic, dynamical, and thermoelectric behavior of layered crystalline phase-change materials. We perform calculations on the pseudobinary compounds $(\mathrm{GeTe})_{x} /\left(\mathrm{Sb}_{2} \mathrm{Te}_{3}\right)$ (GST) with $x=1,2$, and 3. Since the stable configuration of these compounds remains somehow unsettled, we study one stacking configuration for GST124 $(x=1)$, three for GST225 $(x=2)$, and two for GST326 $(x=3)$. A supercell approach is used to check the dynamical stability of the systems while thermoelectric properties are obtained by solving the Boltzmann transport equation. We report that the most accepted stacking configuration of GST124, GST225, and GST326 have metallic character and for the case of $x=2$ and 3, those are the ones with the lowest energy. However, we find the metallic of GST326 configuration to be dynamically unstable. In general, our values of the Seebeck coefficient and thermal conductivity for compounds with $x=1$ and 2 agree very well with the available experimental data. The small differences that we observe with respect to experimental data are attributed to the disorder that is present experimentally and that we have not taken into account. We do not find a Dirac cone in the electronic band structure of GST225, contrarily to previous reports. We attribute this due to the theoretical strain induced by the choice of the pseudopotential.
\end{abstract}

DOI: 10.1103/PhysRevB.97.245205

\section{INTRODUCTION}

Phase-change materials (PCMs) are nowadays emerging as a new generation of nonvolatile memories due to their rapid and reversible transition between crystalline and amorphous phases $[1,2]$. PCMs also exhibit other unique properties that eventually could lead to further technological applications besides their usage in data storage.

For example, there are reasons to consider PCMs for high-temperature thermoelectric applications. Phase-change materials exhibit a low thermal conductivity $(\kappa)$ with relatively high and adjustable electrical conductivity $(\sigma)$ [3,4]. These two properties are related to the thermoelectric figure of merit $Z T=S^{2} \sigma T \kappa^{-1}$, which quantifies the thermoelectric performance, with $S$ being the Seebeck coefficient. Also, most current PCMs and thermoelectric materials are related through their chemical composition, which involves tellurides.

In this work, we investigate the crystalline structure as well as the electronic, dynamical, and thermoelectric properties of three different quasibinary compounds formed by GeTe and $\mathrm{Sb}_{2} \mathrm{Te}_{3}$. We perform ab initio calculations and report the difference in the thermoelectric properties as a function increasing GeTe concentration. The systems considered are quasibinary materials with chemical formula $(\mathrm{GeTe})_{x}\left(\mathrm{Sb}_{2} \mathrm{Te}_{3}\right)_{1}$ (GST family) with values of $x=1,2,3$.

Interestingly enough, multilayered crystalline systems containing $(\mathrm{GeTe})_{x}\left(\mathrm{Sb}_{2} \mathrm{Te}_{3}\right)_{y}$ have been found to be able to switch between two resistance states upon applying low voltage $[5,6]$.

\footnotetext{
*wilfredo.ibarra@correo.buap.mx
}

The change in resistivity has been attributed to the change in local atomic arrangement at the interface between $\mathrm{GeTe}$ $\left(\mathrm{Sb}_{2} \mathrm{Te}_{3}\right)$ blocks [7-9]. This is why these have been called interfacial phase-change materials (IPCMs) [10].

Even though GST materials have been widely studied due to the mentioned memory storage applications, the stable crystal structure for most of the compositions remains largely debated.

Computational methods. We performed calculations using the Vienna ab initio simulation package (VASP) [11-13], which works in the framework of density functional theory (DFT) $[14,15]$. We used the generalized gradient approximation for the exchange correlation in the Perdew-Burke-Ernzerhof revised for solids version (PBEsol) [16]. The plane-wave expansion was set to $400 \mathrm{eV}$ for the self-consistent field (SCF) calculations. Table I summarizes the settings used in our the calculations for all GST compounds studied. We used the projector augmented-wave potentials [17] within a full relativistic scheme, including Ge $s^{2} p^{2}, \mathrm{Sb} s^{2} p^{3}$, and Te $s^{2} p^{4}$ orbitals as part of the valence shell. These pseudopotentials allowed us to include spin-orbit interactions (SOIs). Studies indicate that the inclusion of $d$ orbitals as part of the valence shell usually affects the interatomic distances. However, ab initio calculations on GST225 indicated that qualitative features are independent of the Te $d$ orbital [18]. During the relaxation, we have imposed that the components of the stress tensor were not higher than $0.2 \mathrm{GPa}$. Forces between atoms were restricted to a maximum of $2.5 \times 10^{-5} \mathrm{eV} / \AA$. The electronic band structures, related density of states, and thermoelectric properties were performed in an ulterior non-SCF calculation with denser grids (see Table I). We have computed thermoelectric properties such as the Seebeck coefficient $(S)$, electronic conductivity $(\sigma / \tau)$, 
TABLE I. The $k$-point grid for the SCF and Non-Self consistent field (NSCF) calculations and the size of the supercell used for the calculation of the second- and third-order IFCs. In parentheses we indicate the $k$ grid used in the calculations of the forces for the supercell approach.

\begin{tabular}{lcccc}
\hline \hline \multicolumn{4}{c}{ VASP settings } \\
\hline Compound & $k$ grid & NSCF $k$ grid & Second order & Third order \\
\hline GST124 & $6 \times 6 \times 6$ & $20 \times 20 \times 20$ & $3 \times 3 \times 3\left(2^{3}\right)$ & $2 \times 2 \times 2\left(2^{3}\right)$ \\
GST225 & $8 \times 8 \times 2$ & $32 \times 32 \times 08$ & $5 \times 5 \times 2(\Gamma)$ & $4 \times 4 \times 2(\Gamma)$ \\
GST326 & $4 \times 4 \times 4$ & $16 \times 16 \times 16$ & $2 \times 2 \times 2\left(2^{3}\right)$ & \\
\hline \hline
\end{tabular}

and power factor $\left[S^{2}(\sigma / \tau)\right]$ by solving the Boltzmann transport equations within the constant relaxation-time approximation (CRTA) as implemented in the BOLTZTRAP code [19]. The doping levels were set by the rigid band approximation while the temperature dependence on these bands was neglected.

We have obtained the second-order interatomic force constants through the finite displacement method and using supercells. Due to the large number of atoms in the supercell, these calculations were performed without the inclusion of SOI. We found that the heat capacity shows no dependence on the spinorbit coupling. The SHENGBTE code [20] allowed us to compute the lattice thermal conductivity by solving the Boltzmann transport equation for phonons iteratively. This code goes beyond the relaxation-time approximation which allows a good representation of systems where three-phonon processes are relevant. To obtain the thermal conductivity, we calculated second- and third-order interatomic force. The supercell approach allowed us to get the third-order interatomic force constants (IFCs) with interactions up to the fourth neighbor.

Finally, we have performed calculations for GST124 with the inclusion of van der Waals interaction to compare with our choice of exchange functional. We have relaxed the structure and performed phonon calculations at the $\Gamma$ point for GST124 with three different types of computation schemes for the van der Waals interactions: the Grimme (DFT-D2) nonself-consistent approach, and two self-consistent functionals, Opt86b-vdW and vdW-DF2 [21-25].

The stable configuration of GST multilayer compounds with various concentrations is still a subject of debate [26-28]. Figure 1 shows the most accepted stacking sequence for Ge$\mathrm{Sb}$-Te compounds [29]. Atomic layers are stacked along the $c$ axis (in the hexagonal representation for the case of $x=1$ and 3) with Te-Te adjacent layers surrounded by $\mathrm{Sb}$ atoms. As in previous theoretical studies, we consider alternative stacking sequences for different concentrations of GeTe. In contrast with previous studies, we do not limit our calculations to the energy difference between configurations, but we also compute dynamical properties (phonons) to test the stability of the systems. As far as we know, this is the first systematic study of phonons for the stable crystal structure of $(\mathrm{GeTe})_{x}\left(\mathrm{Sb}_{2} \mathrm{Te}_{3}\right)_{1}$ with $x=1,2$, and 3 .

\section{II. $(\mathrm{GeTe})_{1}\left(\mathrm{Sb}_{2} \mathrm{Te}_{3}\right)(\mathrm{GST124})$}

Crystal structure and lattice parameters. The pseudobinary GST compound noted GST124 $(x=1)$ is a well-known phase-

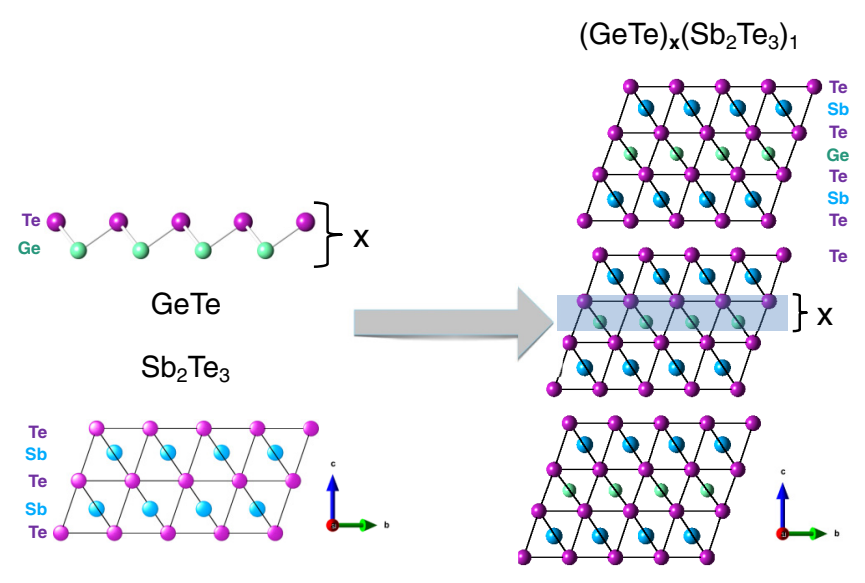

FIG. 1. Representation of the most accepted stacking arrangement of GST compounds. The green, blue, and purple balls represent $\mathrm{Ge}, \mathrm{Sb}$, and Te atoms. In this configuration, the Te-Te adjacent layers are surrounded by antimony atoms.

change material. Similarly to other GST compounds (such as GST225; $x=2$ ), GST124 exhibits two (meta)stable configurations. The stable one is rhombohedral while the metastable structure is rocksaltlike. The transition between metastable and stable configurations is due to diffusion of vacancies $[4,30]$. In the phase-change process, the fast recrystallization drives the system into the metastable crystalline phase. In their experimental work, Matsunaga and Yamada [28] showed that the phase transition to the stable rhombohedral phase occurs at $500 \mathrm{~K}$. Once the system has reached the rhombohedral phase, it remains stable in a range of temperatures between $90 \mathrm{~K}$ and the melting point $(973 \mathrm{~K})$. Our work focuses on the properties of the stable rhombohedral cell. This structure belongs to the space group No. $166(R \overline{3} m)$ with seven atoms in the primitive cell. We explore the stacking configuration shown in Fig. 1. This configuration was proposed by Kooi and De Hosson (in what follows we will name this configuration as KH structure) [27]. Figure 2 shows the primitive rhombohedral cell (a), the nonprimitive hexagonal representation of GST124 (b), as well as its Brillouin zone (c).

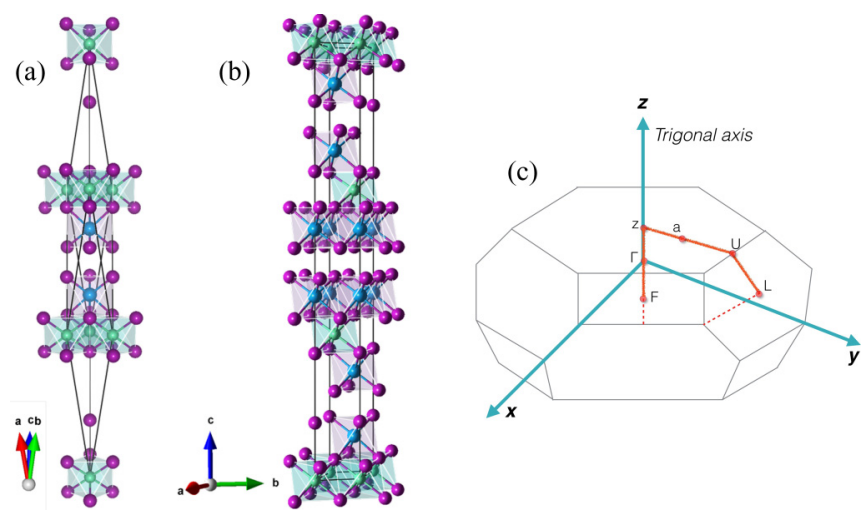

FIG. 2. (a) Rhombohedral primitive cell (extended representation of atoms to illustrate the layered nature of the system). (b) Hexagonal representation with three formula units per cell. (c) First Brillouin zone with high-symmetry points. 
TABLE II. Parameters of the GST124 crystalline structure. Lattice parameters and bond lengths are in $\AA$ and angles are in degrees. Atoms are located at fractional coordinates $(u, u, u)$ and $(1-u, 1-$ $u, 1-u)$ in the primitive rhombohedral structure. The experimental parameter " $g$ " is the percentage of occupancy at such site.

\begin{tabular}{cccc}
\hline \hline \multicolumn{2}{c}{ Lattice parameters } & Expt. [30] & Expt. [27] \\
\hline$a$ & 13.65 & 14.11 & 13.75 \\
$\alpha$ & 17.85 & 17.41 & 17.61 \\
& \multicolumn{3}{c}{ Atomic positions } \\
\hline
\end{tabular}

\begin{tabular}{|c|c|c|c|c|c|}
\hline \multirow[b]{2}{*}{ Site } & \multicolumn{2}{|c|}{ This work } & \multicolumn{3}{|c|}{ Experimental data [30] } \\
\hline & Atom & $u$ & $u$ & Atom & $g$ \\
\hline 3(a) & $\mathrm{Ge}$ & 0 & 0 & $\mathrm{Ge} / \mathrm{Sb}$ & $0.493 / 0.507$ \\
\hline $6(c)$ & $\mathrm{Te}$ & 0.133 & 0.133 & $\mathrm{Te}$ & 1 \\
\hline $6(c)$ & $\mathrm{Te}$ & 0.292 & 0.290 & $\mathrm{Te}$ & 1 \\
\hline $6(c)$ & $\mathrm{Sb}$ & 0.424 & 0.427 & $\mathrm{Ge} / \mathrm{Sb}$ & $0.253 / 0.747$ \\
\hline \multicolumn{3}{|c|}{ Bond lengths } & \multicolumn{3}{|c|}{ Atomic angles } \\
\hline & $-\mathrm{Te}$ & 2.959 & $\mathrm{Ge}-\mathrm{Te}-\mathrm{Ge}$ & & 55.11 \\
\hline & $-\mathrm{Te}$ & $2.99-3.16$ & $\mathrm{Sb}-\mathrm{Te}-\mathrm{Sb}$ & & 54.83 \\
\hline & $\mathrm{Te}$ & 3.669 & & & \\
\hline
\end{tabular}

$A b$ initio calculations carried out with the PBEsol exchange (xc) functional tend to overestimate the lattice parameters but the agreement with experiments is usually better than with PBE. In this work we have not included long-range dispersion forces; instead, we have chosen to use PBESol exchange-correlation functional to improve accuracy on the lattice parameters. It has been shown that negative frequencies reported for GST 225 become positive once the overestimation of the $c$ parameter is reduced closer to experimental values [31]. Here we report that the use of the PBEsol xc functional improves the agreement of lattice parameters of GST225 with respect to experimental measures, which leads to similar results as those obtained by Campi et al. through van der Waals interactions [31]. Table II summarizes our relaxed structure parameters together with the available experimental data. Regarding the atomic bonds, we obtain two different values of the Sb-Te length ( 2.99 and $3.16 \AA$ ) which agrees with the bond-length spread reported experimentally for GST225 [28].

Electronic and dynamic properties. In the KH configuration, we find that GST124 is a semiconductor with a theoretical band gap of $0.225 \mathrm{eV}$ (Fig. 3). Our band-gap value is half of that measured experimentally $(0.55 \mathrm{eV})$ [32]. We find that this forbidden gap is direct and located on the high-symmetry line $Z-\Gamma$, where $Z$ is located at the top of the Brillouin zone [see Fig. 2(c)]. Even though most of the important features that characterize a good thermoelectric material are found in the vicinity of the gap, it has been shown that the systematic underestimate of the gap by DFT does not affect the results for the Seebeck coefficient [33]. The fact that KH-GST124 is found to be a semiconductor agrees with what has been reported theoretically for GST225 with a similar stacking sequence [27]. However, experimental measurements of the change in the resistivity with temperature indicate a metallic character. The works of Shelimova et al. [34] and Siegrist et al. [3] agree that the electronic resistivity of GST124

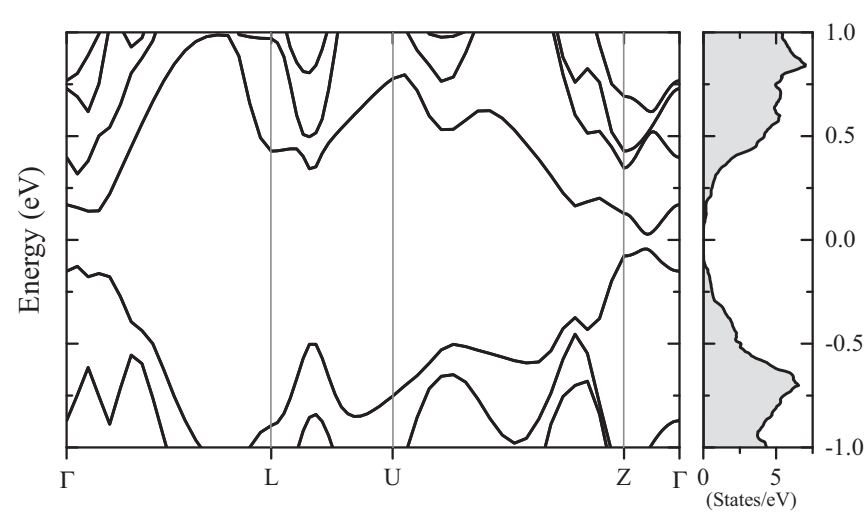

FIG. 3. Calculated electronic band structure and corresponding electronic density of states for GST124. The high-symmetry points are shown in Fig. 2.

increases with increasing of temperature. This result suggests that GST124 is a degenerate semiconductor. The latter is a concept used by Lee et al. [35] to describe GST225 after they found that the number of carriers remain at $10^{19}-10^{20}$ even at $5 \mathrm{~K}$. They mentioned that a degenerate semiconductor is a semiconductor in which the Fermi level lies within the valence band, which originates metalliclike transport properties.

Since the stacking configuration in this compound is still debated, we calculate the dynamic stability of the system through the supercell approach. We use the PHONOPY [36] code to extract the IFCs and with these, we calculate and plot the phonon density of states (PDOS). We attribute the peak observed at slightly negative frequencies $\left(-7 \mathrm{~cm}^{-1}\right.$ at $\Gamma)$ to the numerical accuracy due to the size of the supercell rather than to an actual instability. We relaxed the structures with such tolerance that the maximal residual force is lower than $2.5 \times 10^{-5} \mathrm{eV} / \AA$ for the PBEsol exchange-correlation functional. This does not affect the values of thermoelectric properties presented hereafter. Moreover, we can observe in Table III that the minimal phonon frequency at the $\Gamma$ point is $0.012 \mathrm{~cm}^{-1}$. The results shown in Table III were obtained

TABLE III. Cell volume, lattice parameter and angle, atomic bond lengths, angle between atoms, and minimum and maximum frequencies $\left(\mathrm{cm}^{-1}\right)$ at the $\Gamma$ point for GST124 calculated with different approximations. All the calculations were done without the inclusion of spin-orbit interactions.

\begin{tabular}{lcccc}
\hline \hline & PBEsol & DFT-D2 & Opt86B-vdW & VdW-DF2 \\
\hline Volume $\left(\AA^{3}\right)$ & 208.88 & 211.94 & 216.48 & 249.79 \\
$a(\AA)$ & 13.65 & 13.98 & 13.86 & 14.51 \\
$\alpha(\mathrm{deg})$ & 17.85 & 17.34 & 17.76 & 17.81 \\
$\mathrm{Ge}-\mathrm{Te}(\AA)$ & 2.958 & 2.952 & 2.996 & 3.150 \\
$\mathrm{Sb}-\mathrm{Te}(\AA)$ & $2.99-3.15$ & $2.98-3.15$ & $3.01-3.19$ & $3.13-3.35$ \\
$\mathrm{Te}-\mathrm{Te}(\AA)$ & 3.645 & 3.842 & 3.724 & 3.967 \\
Te-Ge-Te & $88.51-91.49$ & $88.85-91.48$ & $88.79-91.21$ & 91.04 \\
Te-Sb-Te & $84.53-92.54$ & $84.02-92.99$ & $84.45-92.46$ & $84.37-91.71$ \\
Min & 0.012 & 0.011 & 0.0182 & 0.008 \\
Frequency & & & & \\
Max & 172.059 & 175.746 & 168.863 & 147.340 \\
Frequency & & & & \\
\hline \hline
\end{tabular}




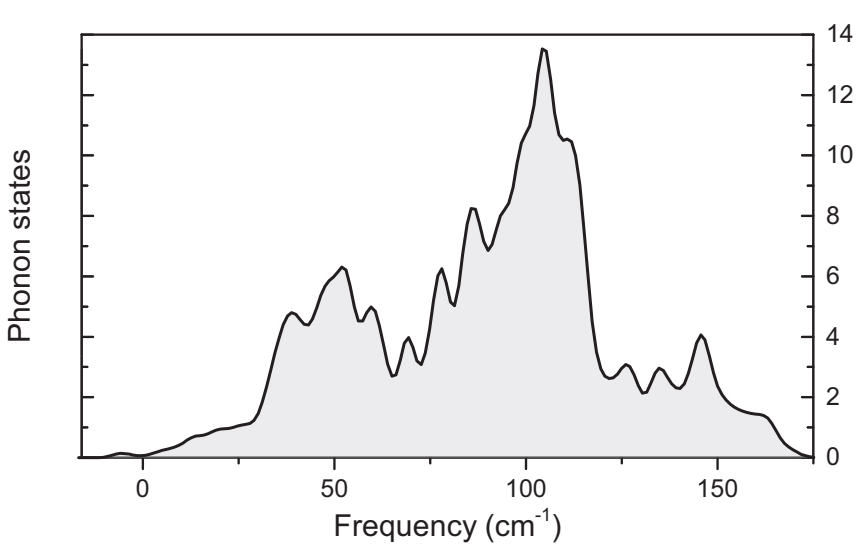

FIG. 4. Phonon density of states for the stable configuration of GST124 obtained with a $3 \times 3 \times 3$ supercell using the finite displacement method.

with the same parameters as in Fig. 4; the only difference is that the value of $0.012 \mathrm{~cm}^{-1}$ was obtained with the primitive cell and density functional perturbation theory (DFPT) [37]. Figure 4 shows the phonon DOS which is similar to the one obtained for GST225 in the Kooi and De Hosson (KH) stacking sequence. In these PDOS, the states increase almost linearly up to $33 \mathrm{~cm}^{-1}$ and the maximum is reached around $100 \mathrm{~cm}^{-1}$. As we mentioned before, Campi et al. [31] show that the inclusion of vdW corrections in GST225 leads to only positive phonons at the $\Gamma$ point. In comparison with our results, the negative frequency shown in the mentioned work was around $30 \mathrm{~cm}^{-1}$. However, with the aim to strengthen our point, we have carried out calculations with different corrections for the $\mathrm{vdW}$ interaction. We show in Table III that not only the phonon frequencies at the $\Gamma$ point are similar between the different corrections and our choice of exchange functional (with the exception of vdW-DF2), but also, the lattice parameter and atomic bond lengths. In general, we observe an increase in the Te-Te bond length when we apply the correction with respect to PBEsol. The vdW-DF2 correction leads to the larger Te-Te bond length and to the larger difference in the maximum phonon frequency with respect to our choice of xc functional.

Thermoelectric properties. The nature and density of carriers are important variables to consider before comparing calculated thermoelectric properties with experimental measures. It has been theoretically predicted that GST124 can be doped either with electrons ( $n$ type) or holes ( $p$ type) [38]. Experimental results show that the carrier concentration of GST124 is of the order of $(2-3) \times 10^{20} \mathrm{~cm}^{-3}$ [34]. The positive values of the Seebeck coefficient indicate that the majority carriers are holes [34,39]. As far as we know, there is no experimental evidence that GST124 could be an $n$-type semiconductor. However, the theoretical work carried out by Sun et al. [38] suggests that, through doping, GST124 could exhibit $n$-type character. Therefore, we plot the carrier concentration dependence of the Seebeck coefficient at different temperatures [Fig. 5(a)] for both $n$ - and $p$-type carriers. This graph indicates that the Seebeck coefficient could be optimized through doping. For example, at room temperature and with a carrier concentration $p=5 \times 10^{19} \mathrm{~cm}^{-3}$, the value of $S$ is
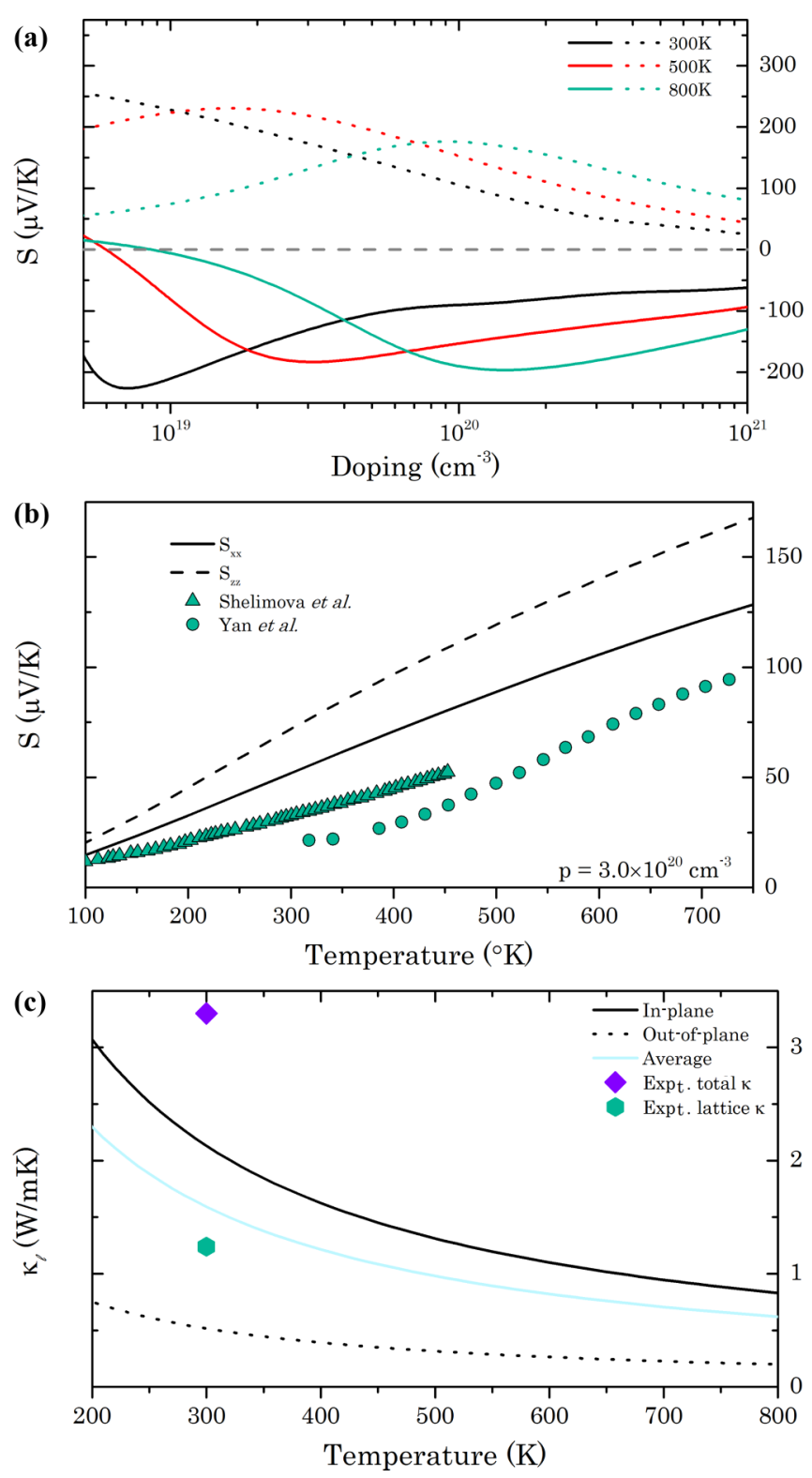

FIG. 5. GST124 (a) Dependence of the trace of the Seebeck coefficient at three different temperatures upon doping with electrons (solid lines) and with holes (dashed lines). (b) Temperature dependence of the in-plane (solid line) and out-of-plane (dashed line) Seebeck coefficient at fixed carrier concentration. The experimental data were extracted from Refs. [34,39]. (c) Temperature dependence of the components of the lattice contribution to the total thermal conductivity as well as the average of the trace of the thermal conductivity tensor and the experimental data. Experimental points were extracted from the work of Konstantinov et al. [40]. The lattice contribution to the thermal conductivity reported in experiments was obtained using the Wiedemann-Franz law.

five times larger than that reported experimentally (at $p=$ $2 \times 10^{20} \mathrm{~cm}^{-3}$ ) [34].

Due to the symmetry of the system, there are two different values for the Seebeck coefficient: one which is degenerate in-plane $\left(S_{x x}\right)$ and one out-of-plane $\left(S_{z z}\right)$. In Fig, 5(b) we show the temperature dependence of the Seebeck coefficient at a fixed carrier concentration value. We also include available 
experimental results for comparison. Even though our theoretical results overestimate the experimental measures, we are able to reproduce the overall trend with temperature. We assume that this overestimation could come from experimental variability since it is possible to find different phases and orientations within the same sample [3]. Even the two experimental measures quoted here differ from each other in a range of temperatures between 300 and $450 \mathrm{~K}$. Nevertheless, our calculations confirm the experimental observation that the Seebeck coefficient does not reach a maximum below $750 \mathrm{~K}$. This implies that this compound could be potentially suitable to be used at very high temperature for thermoelectric energy conversion.

Figure 5(c) shows the lattice contribution to the thermal conductivity. Our calculations agree within $30 \%$ with the experimental values extracted using the Wiedemann-Franz law. The overestimate of the theoretical value was expected since many different minor scattering sources, i.e., impurities, defects, residual isotopic disorder, among others, contribute to the experimental value [20]. Our calculations not only provide information about individual components of the thermal conductivity tensor, but also allow one to explore the nature of the interactions involved. We find that the iterative solution of the BTE is 14\% larger than that obtained with the relaxation-time approximation (iteration zero in SHENGBTE). Li et al. [20] report that for pure $\mathrm{Si}$, the converged value is just $4 \%$ larger than that obtained within RTA. This means that phonon scattering in GST124 is dominated by umklapp processes, but the contributions from normal processes are significant. Finally, we found that the mentioned umklapp processes dominate almost completely in the out-of-plane component of the lattice thermal conductivity.

\section{III. $(\mathrm{GeTe})_{2}\left(\mathrm{Sb}_{2} \mathrm{te}_{3}\right)(\mathrm{GST225})$}

Crystal structure and lattice parameters. Among all phasechange materials, GST225 stands as the most promising PCM for nonvolatile memory devices in terms of transition speed and stability [41]. Moreover, recently it has been claimed that GST225 in the stable configuration exhibits topologicalinsulator behavior [1,42]. In turn, this configuration has been suggested to be the one providing the high-conductivity state in the interfacial phase-change memory device [5].

The stable crystal structure of GST225 is rhombohedral with space group No. 164 and nine atoms per primitive cell. The atoms are stacked along the $c$ axis; however, the sequence of the stacking remains somehow unsettled. Petrov et al. proposed the sequence Te-Sb-Te-Ge-Te-Te-Ge-Te-Sb- (Petrov) [26]. More recently, Kooi and De Hosson proposed an alternative stacking, where $\mathrm{Ge}$ and $\mathrm{Sb}$ atoms interchange positions in the Petrov configuration (KH) [27]. The last proposed stacking sequence is called inverted Petrov (i-Petrov) [43], which consists of an interchange of adjacent Te and Ge atoms in the Petrov structure (Te-Sb-Te-Te-Ge-Ge-Te-Te-Sb-). The three proposed stacking sequences are shown in Fig. 6, and Table IV summarizes the values of the lattice parameters, bond lengths, and energies. We must remark that, as well as in the case of GST124, experimental measures suggest that disorder in the $\mathrm{Ge} / \mathrm{Sb}$ sublattice is also present in GST225 [28]. We have not included such disorder since it is out of the scope of this work.

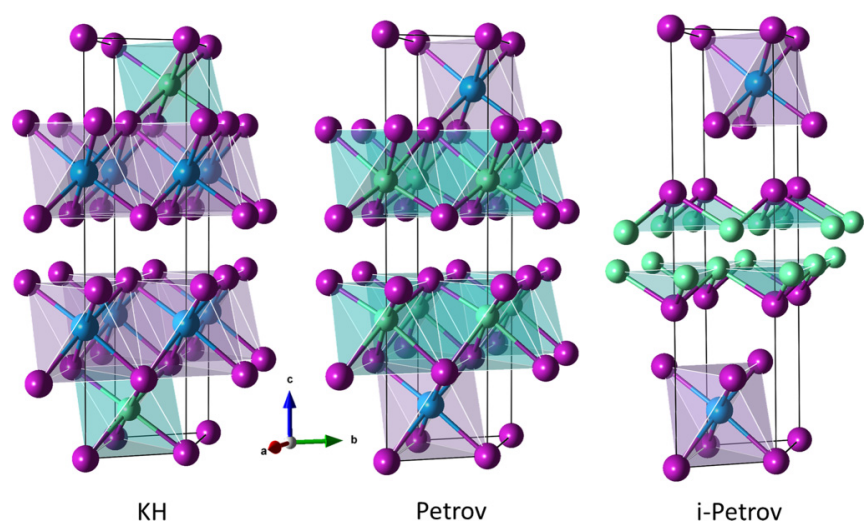

FIG. 6. Primitive rhombohedral representation of GST225 for the Kooi and De Hosson (left), the Petrov (center), and the inverted-Petrov (right) stacking configurations. The colors of the atoms are the same as in Fig. 1.

Our calculations show that the three proposed configurations are very close in energy. Therefore, it is reasonable to think that any of them could be present at ambient conditions. Regarding the structural properties, our results agree with the available experimental data for the three structures. However, there are discrepancies in the Ge-Te bond length. We found that the $\mathrm{KH}$ structure has the lowest energy, therefore, this should be the most stable configuration. However, the Ge-Te bond length is almost a constant $(\delta=0.02 \AA)$, which contrasts with experimental measurements where the Ge-Te bond length ranges from 2.89 to $3.19 \AA$. This spread is also present in the binary compound GeTe in which a short-long bond-length alternation comes from the Peierls distortion mechanism [44]. We found that while in the KH structure the short-long bonds are not present, in the Petrov configuration the difference between those is $0.38 \AA$, in agreement with previous theoretical reports $[18,45]$. This suggests that the Petrov structure is a closer match to the experimental structure. All other computed bond lengths agree very well with the available data, even for the Te-Te bond. The fact that we did not take into account dispersion forces and still get an accurate distance of the

TABLE IV. Energy difference, lattice parameters, and atomic bond lengths for the three proposed stacking configurations in GST225.

\begin{tabular}{|c|c|c|c|c|}
\hline & \multicolumn{4}{|c|}{ Stacking } \\
\hline & $\mathrm{KH}$ & Petrov & i-Petrov & \\
\hline \multirow{2}{*}{$\begin{array}{l}\Delta E(\mathrm{meV} / \text { atom }) \\
\quad \text { Lattice parameters }(\AA)\end{array}$} & 0 & 15.92 & 19.13 & \\
\hline & & & & Ref. [28] \\
\hline \multirow{3}{*}{$\begin{array}{l}\bar{a} \\
c\end{array}$} & 4.23 & 4.2 & 4.16 & 4.22 \\
\hline & 16.88 & 17.14 & 17.59 & 17.24 \\
\hline & \multicolumn{4}{|c|}{ Bond length (§) } \\
\hline $\mathrm{Ge}-\mathrm{Te}$ & \multicolumn{3}{|c|}{$2.95-2.972 .81-3.172 .8$} & $2.89-3.19$ \\
\hline $\mathrm{Sb}-\mathrm{Te}$ & \multicolumn{3}{|c|}{$2.99-3.163 .00-3.142 .98-3.13$} & $32.89-3.19$ \\
\hline $\mathrm{Te}-\mathrm{Te}$ & 3.65 & 3.65 & 3.64 & 3.75 \\
\hline $\mathrm{Ge}-\mathrm{Ge}$ & & & 2.95 & \\
\hline
\end{tabular}


(a)
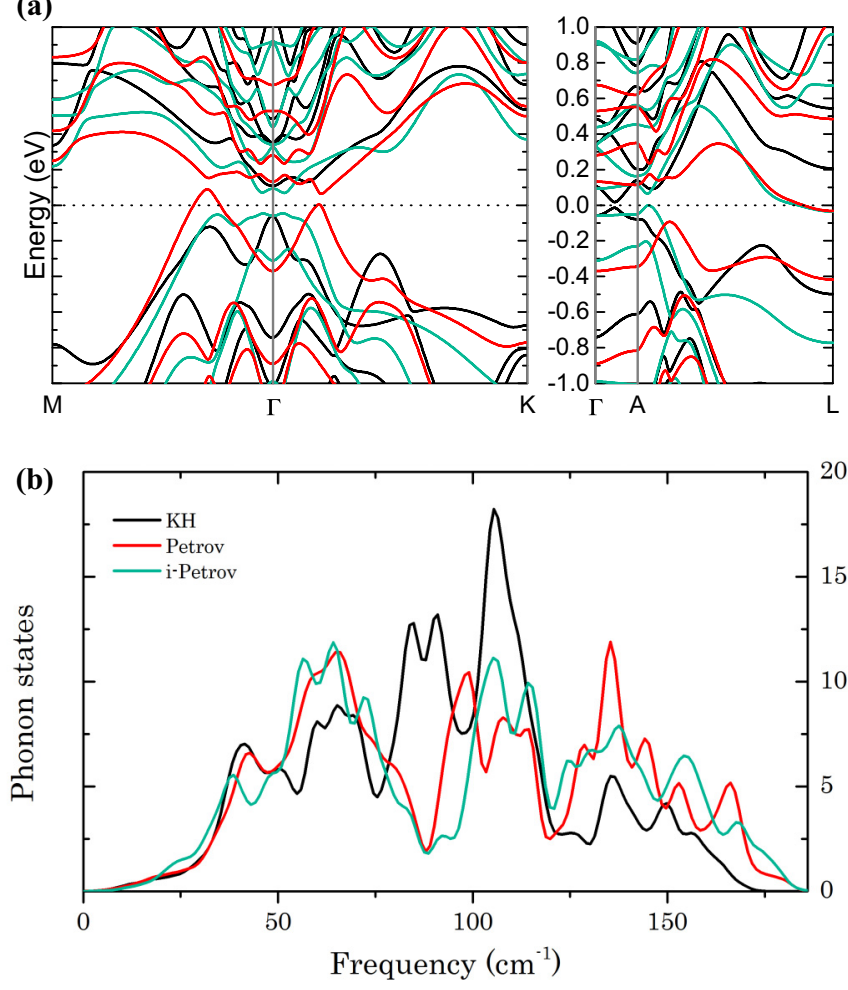

FIG. 7. GST225 (a) Electronic band structure along highsymmetry lines for the proposed stacking configurations. (b) Phonon density of states for the three stacking configurations. Code color is the same for both panels.

interlayer suggests that the Te-Te layers are, at least, partially bonded by covalent interactions [46].

Electronic and dynamic properties. Figure 7(a) shows the electronic band structure of the three different stacking configurations. Our results agree with theoretical references [18] and [45] that the stacking sequence proposed by Kooi and De Hosson (KH) has semiconductor behavior with an electronic band gap of $250 \mathrm{meV}$. The other two structures exhibit a metallic character with bands crossing the Fermi level at different points of the Brillouin zone. Experimentally, the band gap of GST225 is around $500 \mathrm{meV}$, which is double of what we obtain in our DFT calculation. It is well known that DFT systematically underestimates the electronic gap. However, the shape of the bands is, in general, well represented and the effect of the reduced gap in the thermoelectric properties is not significant [33]. In agreement with previous theoretical works $[1,43,47]$, we show that the gap in the KH structure is direct. Nevertheless, the top of the valence band and the bottom of the conduction band lie on the $\Gamma$ - $A$ high-symmetry line. Our results differ from other theoretical calculations in the electronic topology of the i-Petrov stacking sequence. We found no indication of the existence of the mentioned Dirac cone in the band structure [43,47]. We suggest that the Dirac cone could be related to a specific strain in the crystal structure of GST225. We find that the choice of PBE instead of PBEsol induces a theoretical pressure of $-2.2 \mathrm{GPa}$ with respect to the experimental $c$ parameter. Such values of pressure applied to an intrinsic anisotropic semiconductor, such as $\mathrm{Bi}_{2} \mathrm{Te}_{3}$, could lead to electronic topological transitions [33]. Moreover, it has been recently reported that pressure can induce a transition from insulator to gapless Weyl semimetallic state, and a further increase of pressure reverts the transition [48].

On the other hand, experiments indicate that the temperature dependence of the conductivity in GST225 is typical for metals [49]. This suggests that $\mathrm{Ge}_{2} \mathrm{Sb}_{2} \mathrm{Te}_{5}$ is a degenerate semiconductor. Lee et al. also show that at $5 \mathrm{~K}$ the carrier concentration of GST225 is around $2.7 \times 10^{20} \mathrm{~cm}^{-3}$. The authors suggest that this large carrier concentration at low $T$ is because the Fermi level lies inside the valence band [35].

Even though the Petrov and i-Petrov structures have a metallic character, the electronic topologies are different, especially in the vicinity of the Fermi level [zero energy in Fig. 7(a), which is where most of the phenomena related to transport occur. On the other hand, Sosso et al. report that the $\mathrm{KH}$ structure (the one with the lowest energy) has a negative phonon at $\Gamma$ [45]. The negative frequency of the phonon is $-9 \mathrm{~cm}^{-1}$, a very small value that, according to the authors, can be easily stabilized at room temperature. We found out that using PBE pseudopotentials with a small variation of the volume (closer to experimental values), the negative phonon becomes positive. This is one of the reasons that justify our choice for the PBESol xc functional. Our result highlights the strong correlation between the lattice parameters and the phonon modes. While the inclusion of spin-orbit interaction (SOIs) changes the crystal length around $0.05 \%$, the use of PBEsol instead of PBE changes the lattice parameters up to $2.4 \%$. Our work differs from that of Sosso et al. since we perform a dynamical calculation using the supercell approach (see Table I) to ensure that there were no negative phonons in the entire irreducible Brillouin zone. Figure 7(b) shows the phonon density of states of the three configurations. This calculation confirms that all the structures are dynamically stable. The phonon DOS shows a major drop around $90 \mathrm{~cm}^{-1}$ for the Petrov and i-Petrov structures. This reduced phonon density of states will inevitably impact the lattice contribution to the thermal conductivity. See our discussion in the next section where the lattice thermal conductivity is obtained by only taking into account those phonon modes with frequencies below the $90 \mathrm{~cm}^{-1}$. Our results of the $\mathrm{KH}$ structure are similar to those reported in Ref. [50]. Phonon band structure has been reported for some of these structures in the works of Campi et al. [31,51].

Thermoelectric properties. The Seebeck coefficient is a property strongly related to the electronic topology of the system. Since the three configurations have different electronic structures we expect substantial differences in $S$. Two configurations have a metallic behavior with bands crossing the Fermi level at different points in the Brillouin zone. The common metallic nature will lead to small relative values of the Seebeck coefficient. A recent theoretical work carried out by Sun and co-workers show that the low defect formation energies in GST225 originate a high concentration of vacancies that makes GST225 a permanent $p$-type semiconductor [38]. We thus perform calculations on the Seebeck coefficient as a function of the $p$-type carrier concentration at $300 \mathrm{~K}$ for each stacking configuration.

Figure 8(a) shows the computed Seebeck coefficient dependency on the carrier concentration. For the KH stack- 


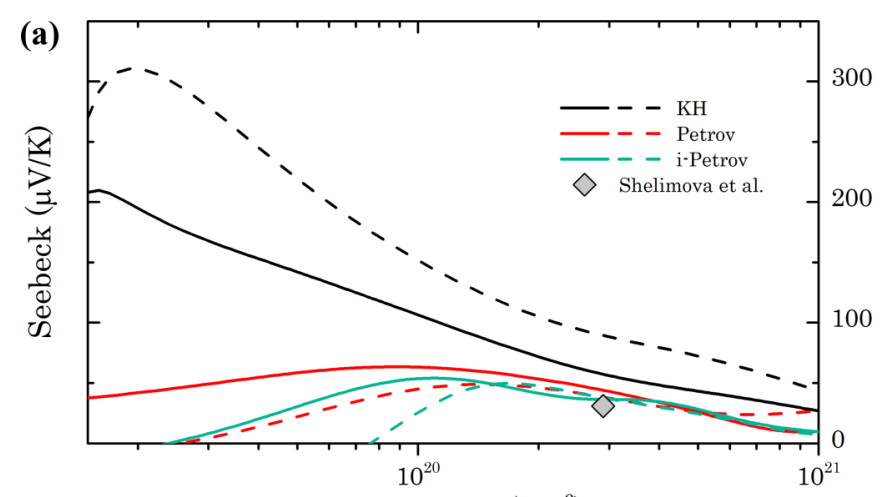

Doping level $\left(\mathrm{cm}^{-3}\right)$
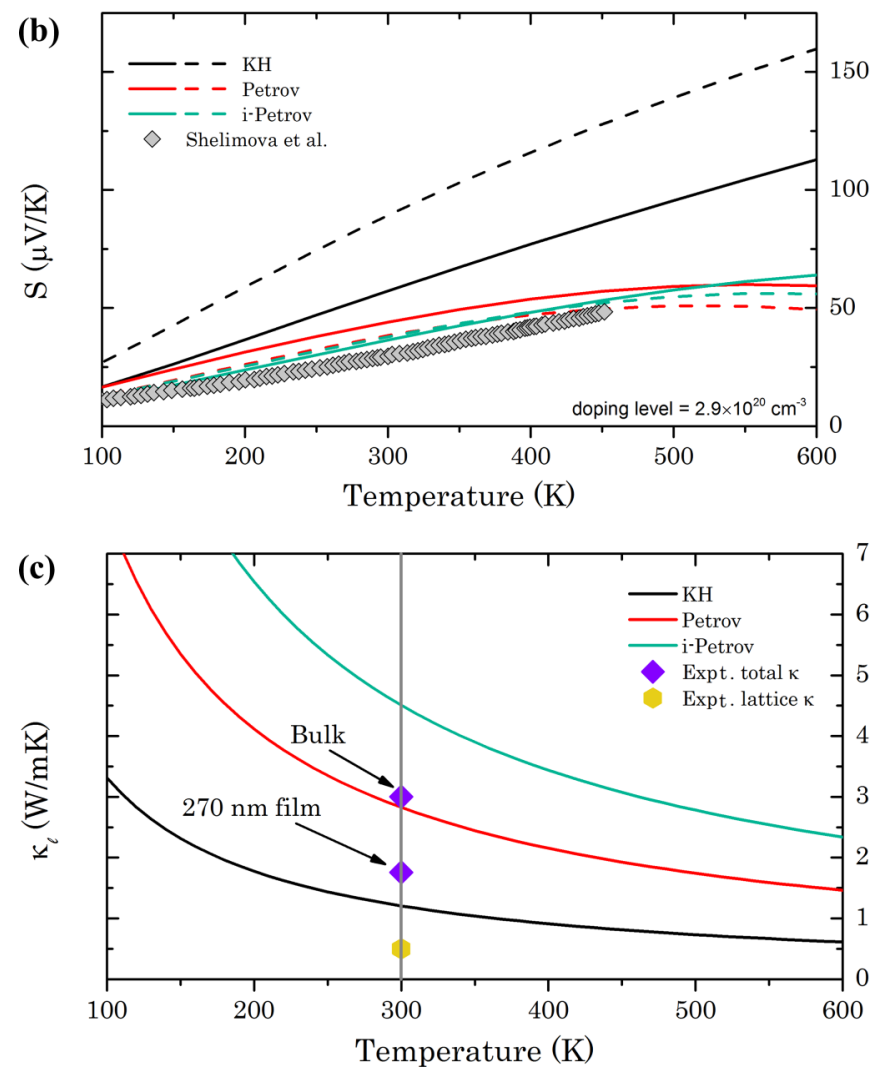

FIG. 8. GST225 (a) Carrier concentration dependence of the Seebeck coefficient at $300 \mathrm{~K}$. Solid and dashed lines denote in-plane and out-of-plane $S$. (b) Temperature dependence of the in-plane (solid) and out-of-plane (dashed) Seebeck coefficient at experimental carrier concentration for the three proposed stacking configurations. Experimental data was extracted from Ref. [34]. (c) Lattice contribution to the total thermal conductivity for the three proposed stacking configurations. Experimental data was extracted from Refs. [34,52].

ing sequence (semiconductor), the values of the Seebeck coefficient vary from $300 \mu \mathrm{V} / \mathrm{K}$ at $2 \times 10^{19} \mathrm{~cm}^{-3}$ down to nearly $25 \mu \mathrm{V} / \mathrm{K}$ at $1 \times 10^{21} \mathrm{~cm}^{-3}$. These values agree with characteristic values in semiconductors such as $\mathrm{PbTe}, \mathrm{Sb}_{2} \mathrm{Te}_{3}$, and $\mathrm{Bi}_{2} \mathrm{Te}_{3}[19,53,54]$. The other two structures with metallic electronic topology, as we expected, exhibit considerably smaller values than those obtained in the KH configuration. However, both stacking configurations Petrov and i-Petrov are in very good agreement with available experimental data while the KH structure slightly overestimates experimental results.
Figure 8(a) also shows that the Seebeck coefficient could be optimized with respect to the carrier density. In the case of the KH structure, the optimal value of doping is around $2 \times$ $10^{19} \mathrm{~cm}^{-3}$. With regard to the metallic structures, the optimal carrier concentration is near to $1.5 \times 10^{20} \mathrm{~cm}^{-3}$. Optimal values of the carrier concentration could eventually double the value of the Seebeck coefficient at room temperature. Nevertheless, the experimental value of the electronic gap suggests that GST 225 could be suitable for a high-temperature thermoelectric application. For this reason, we explore the temperature dependence of the Seebeck coefficient at fixed doping [Fig. 8(b). We impose a carrier density equal to the experimental one reported by Shelimova et al. [34].

Figure $8(\mathrm{~b})$ shows the excellent agreement between our theoretical results and experimental data. The Petrov and i-Petrov structures are those with the closest agreement with the experiment. The semiconducting configuration clearly overestimates the experimental results. Nevertheless, the three structures and experimental data show a continuous increase of the Seebeck coefficient with temperature. This confirms the hypothesis that GST225 would exhibit good thermoelectric performance at temperatures above room temperature. With phase-change materials, there is always a concern about stability of the structure at high temperatures [3]. Therefore, the works from Friedrich et al. [55] and Lyeo et al. [52] make sure that the stable hexagonal phase is reached through high-temperature heating. Independent of any crystalline phase transition considerations, the work of Shelimova et al. [34] shows that there is no abrupt change in Seebeck coefficient with temperatures up to $800 \mathrm{~K}$.

We computed the lattice contribution to the thermal conductivity for the three configurations of GST225. The agreement with the experimental lattice conductivity extracted from the total conductivity by using the Wiedemann-Franz relation is reasonable. We find that the $\mathrm{KH}$ configuration is the one with the lowest thermal conductivity [Fig. 8(c). It has been shown that the overestimation of the lattice thermal conductivity obtained from $a b$ initio calculations can be overcome by the inclusion of an effective disorder on the cation masses [31]. The overestimation was expected since, experimentally, there are many other different sources of scattering that we do not take into account in our calculations. However, the inclusion of an effective $\mathrm{Sb} / \mathrm{Ge}$ disorder together with vacancies, although neglecting all local relaxation effects on the electronic and phonon band structure, leads to values that are very close to the experimental ones. Therefore, we suggest that an explicit inclusion of cation disorder and vacancies in hexagonal GST compounds should be realized, which is beyond the scope of the present paper. Normal processes contribute less in this compound than in GST124 (5.3\% in the in-plane direction and $0.7 \%$ in the out-of-plane direction at $300 \mathrm{~K}$ ). Calculations of $\kappa$ usually neglect the contribution of optical branches due to their small group velocities and frequencies that reside at much higher energy than acoustic branches [56]. However, there are special cases, such as diamond, where scattering processes that involve interaction between acoustic and optical phonons contribute to as much as $80 \%$ of total values [56]. With this in mind, we explore the effect of the quasibipartite phonon structure (characterized by the abrupt drop in the PDOS near to $90 \mathrm{~cm}^{-1}$ ) found in the Petrov and i-Petrov structures. We 
find that phonons with frequencies lower that $90 \mathrm{~cm}^{-1}$ are responsible for the $74.6 \%(97.7 \%)$ of the total lattice thermal conductivity to the in-plane (out-of-plane) direction for the iPetrov configuration at $300 \mathrm{~K}$. Similarly, in the Petrov stacking configuration the contribution of these phonons reaches $81 \%$ and $97.5 \%$ of the total $\kappa_{l}$ in the in-plane and out-of-plane directions, respectively. The KH configuration lacks this bipartite phonon dispersion and possesses the lowest lattice thermal conductivity. According to Carrete et al., the large number of low-frequency optical modes yields low thermal conductivity. Such a bipartite phonon dispersion was found in $\mathrm{SnSe}$ with the Pnma structure [57]. In a previous paper, we have found that the phonon band crossing helps to reduce the thermal conductivity in $\mathrm{Li}-\mathrm{Mg}$ compounds [58].

\section{IV. $(\mathrm{GeTe})_{3}\left(\mathrm{Sb}_{2} \mathrm{Te}_{3}\right)(\mathrm{GST} 326)$}

We further investigate the change of the thermoelectric properties of pseudobinary compounds by increasing the amount of GeTe in the system. In what follows, we present our work for a system with three GeTe formula units for one $\mathrm{Sb}_{2} \mathrm{Te}_{3}$ unit. As we show hereafter, the generally accepted stacking configuration of GST326 is dynamically unstable. Therefore, we have changed the stacking sequence in order to find the arrangement of the atomic layers that yields the dynamic stability of the system. The one with the lowest energy is the unstable one, which is rather paradoxical, and the stacking sequence is -Te-Ge-Te-Ge-Te-Sb-Te-Te-Sb-Te-Ge(named as KH-326). The stacking configuration that leads to the dynamical stability of the system is -Te-Ge-Te-Sb-TeGe-Te-Te-Ge-Te-Sb- (named as Petrov-326). The differences between the two proposed configurations are analogous to those in GST225 between the KH and Petrov structures. Our results of the $c$ parameter $(61.21 \AA)$ are close to that reported by Karpinsky et al. [59] (62.2 ̊) for both stacking configurations. Sa et al. [60] showed that the structure with the lowest energy is the one named as KH-326. The energy difference between the $\mathrm{KH}$ and Petrov structures is only $-0.014 \mathrm{eV} /$ atom which is even smaller than what we have found in GST225 between $\mathrm{KH}$ and Petrov stacking configurations. When the Ge atoms are surrounding the Te-Te adjacent layers the system is metallic. On the other hand, when the Te-Te interstitial layer is surrounded by $\mathrm{Sb}$ atoms, the electronic character becomes semiconducting (bottom, Fig. 9).

As far as we know, this is the first phonon calculation performed on GST326. The negative frequency in the KH configuration reaches $-40 \mathrm{~cm}^{-1}$ at $\Gamma$. This value is large which, given our careful relaxation of the atomic positions, suggests that the system is unstable. However, this is not the only point where we find negative frequencies. The negative mode remains negative on the high-symmetry line that connects the $\Gamma$ and $A$ points [see Brillouin zone in Fig. 2(c). We notice that this optical mode is degenerate, and corresponds to the movement of the Ge and $\mathrm{Sb}$ atoms in phase along the $x$ (or $y$ ) axis while all Te atoms move out of phase in the same direction. Interestingly enough, such movements have been invoked by $\mathrm{Yu}$ and Robertson [7] to explain the electrical switching in IPCMs. However, the switching mechanism in IPCMs involves several complex stages. Even though our calculations suggest that the mentioned arrangement is unstable, we cannot discard
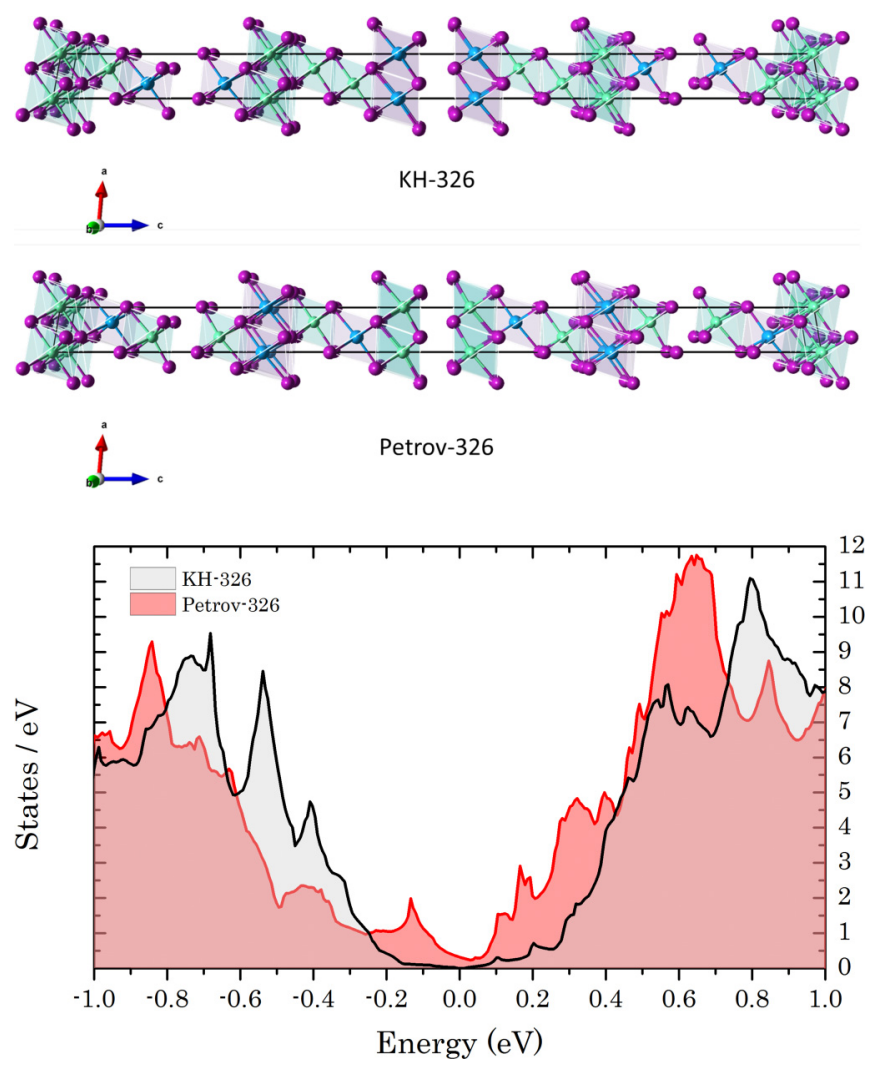

FIG. 9. From top to bottom: Nonprimitive hexagonal representation of the KH-326 and Petrov-326 structures; electronic density of states of the two proposed stacking configurations. (The Fermi level is taken as reference energy.)

the possibility that long-range dispersion forces could lead to a stabilization of GST326, as was observed in GST225 [31,6163]. Therefore, we decided to perform a set of calculations with DFT-D2. We have summarized our results in Table V. We have relaxed the structures and compared energies, volumes, and lattice parameters as well as phonon frequencies at the $\Gamma$ point. Our phonon frequencies in Table $V$ were calculated with DFPT and only at $\Gamma$. We have found that the results obtained

TABLE V. Total energy, volume, atomic bond lengths, lattice parameter, and angle of the two proposed configurations for GST326 with PBEsol and with DFT-D2. Minimum and maximum frequencies are at the $\Gamma$ point and their units are $\mathrm{cm}^{-1}$.

\begin{tabular}{lccccc}
\hline \hline & \multicolumn{2}{c}{ KH-326 } & & \multicolumn{2}{c}{ Petrov-326 } \\
\cline { 2 - 3 } \cline { 5 - 6 } & PBEsol & DFT-D2 & & PBEsol & DFT-D2 \\
\hline Energy $(\mathrm{eV})$ & -46.18 & -45.52 & & -46.02 & -45.38 \\
Volume $\left(\AA^{3}\right)$ & 312.05 & 315.18 & & 312.54 & 317.14 \\
$a(\AA)$ & 20.432 & 20.752 & & 20.594 & 21.038 \\
$\alpha(\mathrm{deg})$ & 11.839 & 11.622 & & 11.706 & 11.489 \\
Ge-Te $(\AA)$ & $2.94-2.98$ & $2.93-2.98$ & & $2.94-2.95$ & $2.80-3.19$ \\
Sb-Te $(\AA)$ & $2.98-3.16$ & $2.98-3.16$ & & $2.99-3.16$ & $2.98-3.15$ \\
Te-Te $(\AA)$ & 3.66 & 3.85 & & 3.62 & 3.83 \\
Min frequency & -30.23 & -34.77 & & -0.06 & -0.14 \\
Max frequency & 171.91 & 173.19 & & 185.63 & 188.60 \\
\hline \hline
\end{tabular}




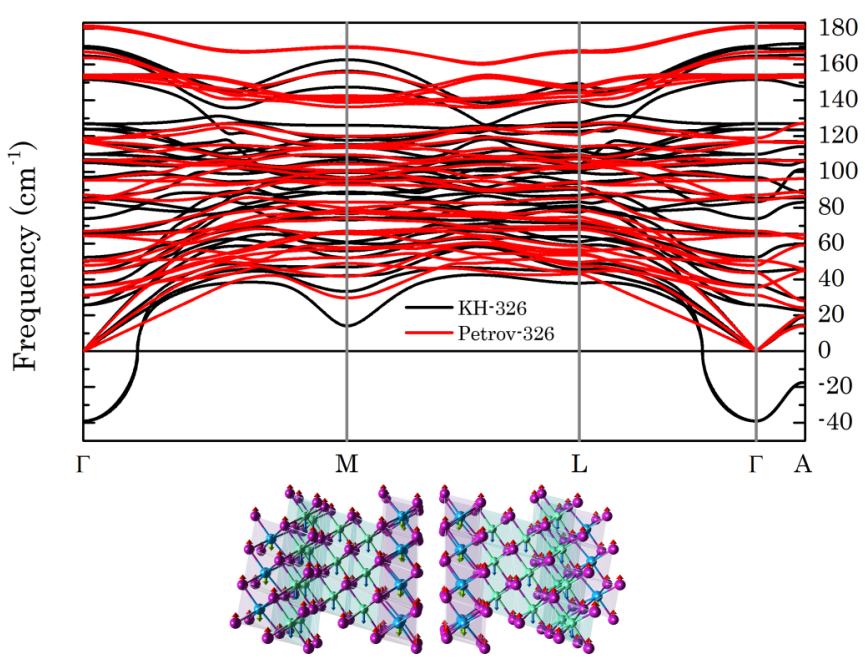

FIG. 10. Phonon band structure for the two stacking sequences. The KH-326 configuration is clearly unstable. The lower panel shows the KH-326 structure with arrows that denote the movement of the atom that produces the negative frequencies.

with PBEsol and DFT-D2 are comparable for both structures. In both cases, the KH-326 structure has lower energy than the Petrov-326; however, KH-326 exhibits dynamical instability (Fig. 10). It can also be noted that DFT-D2 correction increases the volume of the structures around $\sim 1 \%$ due to the increase of the Te-Te bond length.

Thermoelectric properties. Although we have found that KH-326 is unstable, we will show the Seebeck coefficient for both stacking configurations. Experimentally, crystalline GST326 contains more intrinsic vacancies than the two previous compounds, which makes the comparison of our calculations with the experiment difficult, the samples being very much dependent on growing method and thermal history. For example, Sittner et al. [64] measure the temperature dependence of the metastable structure of GST326 treated at different annealing temperatures. They report that a $50^{\circ}$ temperature variation leads to $33 \%$ difference in the Seebeck coefficient. This behavior was attributed to the rearrangement of the intrinsic vacancies. We first compute the doping dependence of the Seebeck coefficient. As expected, the two proposed configurations have different behaviors. The values of $S$ for the Petrov-326 configuration are almost a third of those for $\mathrm{KH}-326$ at all temperatures. This is again due to the fact that KH-326 is semiconducting while Petrov-326 is metallic. Since in GST225 the majority of carriers are holes, we assume that in GST326 a large number of intrinsic vacancies will lead to $p$ type of carriers as well. In agreement with our results for the other compounds, we fix the carrier concentration at $3 \times 10^{20} \mathrm{~cm}^{-3}$. Figure 11(b) shows our results together with the experimental data from Rosenthal et al. [29], that were measured on the metastable cubic phase. However, our results agree nicely with the experiment. No comparison with data measured on the stable GST326 phase is possible as Sittner et al. [64] evidenced a very large variability in the measured Seebeck coefficient with annealing temperature. The strong variation of the $S$ with the annealing $T$ could be due to
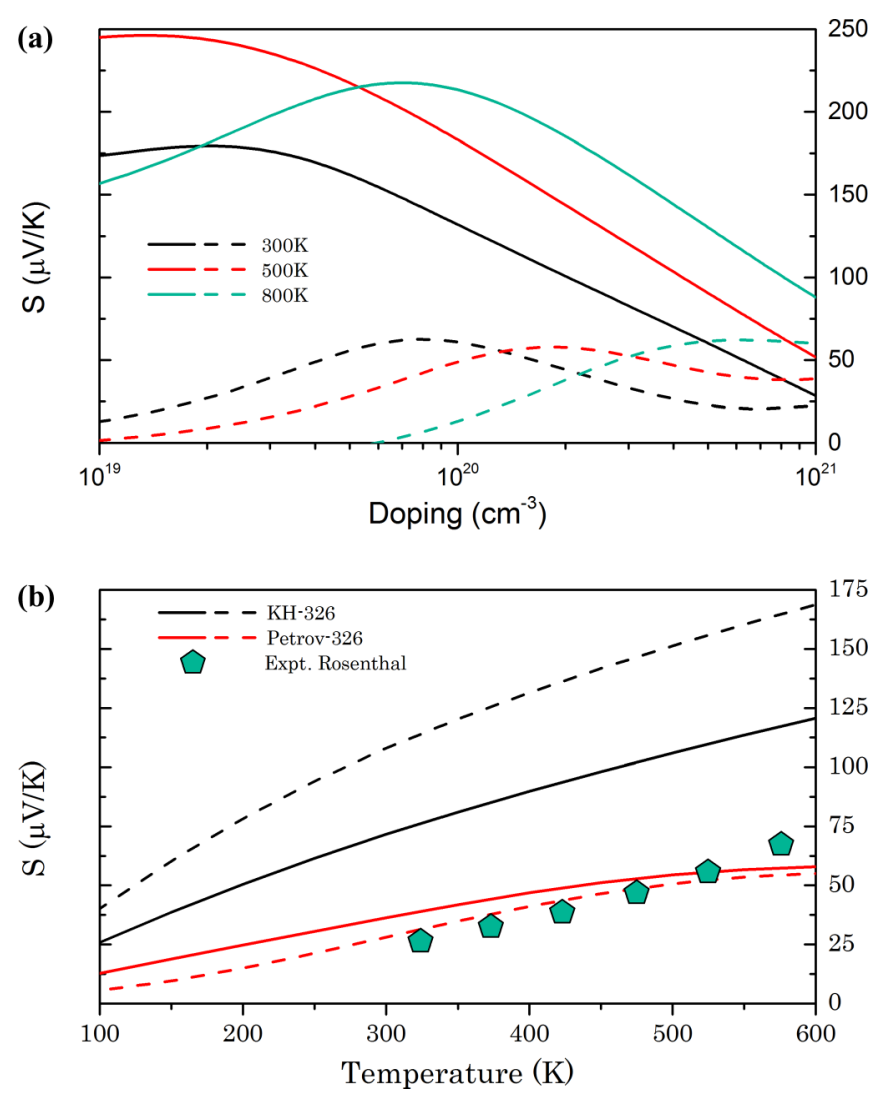

FIG. 11. (a) Carrier concentration dependence of the Seebeck coefficient for GST326. Solid and dashed lines denote values for the KH-326 and Petrov-326 stacking arrangements, respectively. (b) Temperature dependence of the in-plane (solid lines) and out-of-plane (dashed lines) Seebeck coefficients for stacking configurations of GST326. The experimental data were extracted from Ref. [29].

changes in the carrier concentration like in the case of $\mathrm{Bi}-\mathrm{Sb}$ compounds [65].

\section{CONCLUSIONS}

We explored the electronic, dynamic, and thermoelectric properties of three pseudobinary compounds $(\mathrm{GeTe})_{x} /\left(\mathrm{Sb}_{2} \mathrm{Te}_{3}\right)_{1}$ with $x=1,2,3$. For GST124, our study reveals that the semiconductor structure is dynamically stable. Our values systematically overestimate the experimental results related to the temperature dependence of the Seebeck coefficient. However, we reproduce the overall trend and show that an optimization of $S$ with the carrier concentration could lead to the increase of the thermoelectric performance. Regarding the thermal conductivity, we showed that the decrease of the lattice contribution, together with the increase of the Seebeck coefficient suggests that the maximum thermoelectric performance $(Z T)$ in this compound should be around $600 \mathrm{~K}$. Our theoretical maximum is $Z T=0.83$ at $600 \mathrm{~K}(S=116.43 \mu \mathrm{V} / \mathrm{K} ; k=0.82 \mathrm{~W} / \mathrm{mK}$; $\left.\rho_{\text {exp }}=12 \times 10^{-6} \Omega \mathrm{m}[34]\right)$. However, as we mentioned, our results overestimate the Seebeck coefficient and underestimate the thermal conductivity.

We explored three different stacking configurations of the pseudobinary compound with $x=2$, and found that the 
sequence proposed by Kooi and De Hosson (-Te-Ge-Te-SbTe-Te-Sb-Te-Ge-) has the lowest energy. However, the other two stacking configurations are only higher in energy by 20 $\mathrm{meV} /$ atom. This small energy difference suggests that at room temperature the three proposed stacking configurations could possibly coexist. We also investigated the dynamical stability of the three configurations through our calculations of phonon modes. The $\mathrm{KH}$ configuration has the best thermoelectric performance of the three proposed stacking sequences. We determined that the theoretical Dirac cone in the band structure that has been previously reported only exists under specific conditions of strain in the inverted-Petrov structure.

Finally, we explored the electronic properties and the Seebeck coefficient of GST326 $(x=3)$. We realized that the most accepted stacking sequence of this pseudobinary compound is unstable. Similarly to what we found in GST225, the KH-326 and the Petrov-326 configurations have semiconductor and metallic characters, respectively.

In summary, the phase-change materials that we studied in this work could improve their Seebeck coefficient through optimization of the carrier concentration. Another possibility to improve the thermoelectric performance could be the introduction of disorder, which is expected to produce an increase in the thermal conductivity while the electrical conductivity and the Seebeck coefficient would remain at significant values. Finally, our work suggests further studies to elucidate the stable structure of GST326.

\section{ACKNOWLEDGMENTS}

W.I.H. thankfully acknowledges the computer resources, technical expertise, and support provided by the Laboratorio Nacional de Supercómputo del Sureste de México and the support from the grant provided by "CONACyT Repatriación 2017." The authors also acknowledge the computational time made available by the Belgian CECI, SEGI-ULg, and PRACE projects NanoTherm (2IP FP7 RI-283493) and ThermoSpin on ARCHER (3IP FP7 RI-312763). J.Y.R. gratefully acknowledges the computational resources provided by the Consortium des Equipements de Calcul Intensif (CECI), funded by the Fonds de la Recherche Scientifique de Belgique (F.R.S.-FNRS) under Grant No. 2.5020.11 and the Tier-1 supercomputer of the Federation Wallonie-Bruxelles, infrastructure funded by the Walloon Region under the Grant Agreement No. 1117545. J.Y.R. acknowledges support from ULg and the Communauté Française de Belgique (ARC AIMED 15/19-09).
[1] J. Kim, J. Kim, and S.-H. Jhi, Phys. Rev. B 82, 201312 (2010).

[2] J.-Y. Raty, C. Otjacques, J.-P. Gaspard, and C. Bichara, Solid State Sci. 12, 193 (2010); morphology and dynamics of nanostructures and disordered systems via atomic-scale modeling.

[3] T. Siegrist, P. Jost, H. Volker, M. Woda, P. Merkelbach, C. Schlockermann, and M. Wuttig, Nat. Mater. 10, 202 (2011).

[4] W. Zhang, A. Thiess, P. Zalden, R. Zeller, P. H. Dederichs, J.-Y. Raty, M. Wuttig, S. Blügel, and R. Mazzarello, Nat. Mater. 11, 952 (2012).

[5] M. Lankhorst, B. Ketelaars, and R. Wolters, Nat. Mater. 4, 347 (2005).

[6] M. Wuttig and N. Yamada, Nat. Mater. 6, 824 (2007).

[7] X. Yu and J. Robertson, Sci. Rep. 5, 12612 (2015).

[8] J. Kalikka, J. Akola, J. Larrucea, and R. O. Jones, Phys. Rev. B 86, 144113 (2012).

[9] J. Momand, R. Wang, J. E. Boschker, M. A. Verheijen, R. Calarco, and B. J. Kooi, Nanoscale 9, 8774 (2017).

[10] R. Simpson, P. Fons, A. Kolobov, T. Fukaya, M. Krbal, T. Yagi, and J. Tominaga, Nat. Nanotechnol. 6, 501 (2011).

[11] G. Kresse and J. Hafner, Phys. Rev. B 47, 558 (1993).

[12] G. Kresse and J. Hafner, Phys. Rev. B 49, 14251 (1994).

[13] G. Kresse and J. Furthmüller, Phys. Rev. B 54, 11169 (1996).

[14] P. Hohenberg and W. Kohn, Phys. Rev. 136, B864 (1964).

[15] W. Kohn and L. J. Sham, Phys. Rev. 140, A1133 (1965).

[16] J. P. Perdew, A. Ruzsinszky, G. I. Csonka, O. A. Vydrov, G. E. Scuseria, L. A. Constantin, X. Zhou, and K. Burke, Phys. Rev. Lett. 100, 136406 (2008).

[17] P. E. Blöchl, Phys. Rev. B 50, 17953 (1994).

[18] G. Lee and S.-H. Jhi, Phys. Rev. B 77, 153201 (2008).

[19] G. K. H. Madsen and D. J. Singh, Comput. Phys. Commun. 175, 67 (2006).

[20] W. Li, J. Carrete, N. A. Katcho, and N. Mingo, Comput. Phys. Commun. 185, 1747 (2014).
[21] S. Grimme, J. Comput. Chem. 27, 1787 (2006).

[22] M. Dion, H. Rydberg, E. Schröder, D. C. Langreth, and B. I. Lundqvist, Phys. Rev. Lett. 92, 246401 (2004).

[23] G. Román-Pérez and J. M. Soler, Phys. Rev. Lett. 103, 096102 (2009).

[24] J. Klimeš, D. R. Bowler, and A. Michaelides, J. Phys.: Condens. Matter 22, 022201 (2010).

[25] J. Klimeš, D. R. Bowler, and A. Michaelides, Phys. Rev. B 83, 195131 (2011).

[26] I. I. Petrov, R. M. Imamov, and Z. G. Pinsker, Sov. Phys. Crystallogr. 13, 339 (1968).

[27] B. J. Kooi and J. T. M. De Hosson, J. Appl. Phys. 92, 3584 (2002).

[28] T. Matsunaga, N. Yamada, and Y. Kubota, Acta Crystallogr., Sect. B: Struct. Sci., Cryst. Eng. Mater. 60, 685 (2004).

[29] T. Rosenthal, M. N. Schneider, C. Stiewe, M. Döblinger, and O. Oeckler, Chem. Mater. 23, 4349 (2011).

[30] T. Matsunaga and N. Yamada, Phys. Rev. B 69, 104111 (2004).

[31] D. Campi, L. Paulatto, G. Fugallo, F. Mauri, and M. Bernasconi, Phys. Rev. B 95, 024311 (2017).

[32] J.-W. Park, S. H. Eom, H. Lee, J. L. F. Da Silva, Y.-S. Kang, T.-Y. Lee, and Y. H. Khang, Phys. Rev. B 80, 115209 (2009).

[33] W. Ibarra-Hernández, M. J. Verstraete, and J.-Y. Raty, Phys. Rev. B 90, 245204 (2014).

[34] L. Shelimova, O. Karpinskii, P. Konstantinov, M. Kretova, E. Avilov, and V. Zemskov, Inorg. Mater. 37, 342 (2001).

[35] B.-S. Lee, J. R. Abelson, S. G. Bishop, D.-H. Kang, B.-k. Cheong, and K.-B. Kim, J. Appl. Phys. 97, 093509 (2005).

[36] A. Togo, F. Oba, and I. Tanaka, Phys. Rev. B 78, 134106 (2008).

[37] X. Gonze and C. Lee, Phys. Rev. B 55, 10355 (1997).

[38] Z. Sun, Y. Pan, J. Zhou, B. Sa, and R. Ahuja, Phys. Rev. B 83, 113201 (2011). 
[39] F. Yan, T. Zhu, X. Zhao, and S. Dong, Appl. Phys. A 88, 425 (2007).

[40] P. Konstantinov, L. Shelimova, E. Avilov, M. Kretova, and V. Zemskov, Inorg. Mater. 37, 662 (2001).

[41] Z. Sun, J. Zhou, and R. Ahuja, Phys. Rev. Lett. 96, 055507 (2006).

[42] B. Sa, J. Zhou, Z. Song, Z. Sun, and R. Ahuja, Phys. Rev. B 84, 085130 (2011).

[43] J. Tominaga, A. V. Kolobov, P. Fons, T. Nakano, and S. Murakami, Adv. Mater. Interfaces 1, 1300027 (2014).

[44] J. Y. Raty, V. Godlevsky, P. Ghosez, C. Bichara, J. P. Gaspard, and J. R. Chelikowsky, Phys. Rev. Lett. 85, 1950 (2000).

[45] G. C. Sosso, S. Caravati, C. Gatti, S. Assoni, and M. Bernasconi, J. Phys.: Condens. Matter 21, 245401 (2009).

[46] A. M. Mio, S. M. S. Privitera, V. Bragaglia, F. Arciprete, C. Bongiorno, R. Calarco, and E. Rimini, Nanotechnology 28, 065706 (2017).

[47] B. Sa, J. Zhou, Z. Sun, J. Tominaga, and R. Ahuja, Phys. Rev. Lett. 109, 096802 (2012).

[48] S. Singh, A. C. Garcia-Castro, I. Valencia-Jaime, F. Muñoz, and A. H. Romero, Phys. Rev. B 94, 161116 (2016).

[49] T. Kato and K. Tanaka, Jpn. J. Appl. Phys. 44, 7340 (2005).

[50] T. Tsafack, E. Piccinini, B.-S. Lee, E. Pop, and M. Rudan, J. Appl. Phys. 110, 063716 (2011).

[51] D. Campi, E. Baldi, G. Graceffa, G. C. Sosso, and M. Bernasconi, J. Phys.: Condens. Matter 27, 175009 (2015).

[52] H.-K. Lyeo, D. G. Cahill, B.-S. Lee, J. R. Abelson, M.-H. Kwon, K.-B. Kim, S. G. Bishop, and B.-k. Cheong, Appl. Phys. Lett. 89, 151904 (2006).
[53] D. J. Singh, Phys. Rev. B 81, 195217 (2010).

[54] T. Thonhauser, T. J. Scheidemantel, J. O. Sofo, J. V. Badding, and G. D. Mahan, Phys. Rev. B 68, 085201 (2003).

[55] I. Friedrich, V. Weidenhof, W. Njoroge, P. Franz, and M. Wuttig, J. Appl. Phys. 87, 4130 (2000).

[56] N. Mingo, D. A. Stewart, D. A. Broido, L. Lindsay, and W. $\mathrm{Li}$, in Length-Scale Dependent Phonon Interactions, Topics in Applied Physics Vol. 128, edited by G. P. S. Subhash and L. Shind (Springer, New York, 2014), pp. 137-173.

[57] J. Carrete, N. Mingo, and S. Curtarolo, Appl. Phys. Lett. 105, 101907 (2014).

[58] O. Pavlic, W. Ibarra-Hernández, I. Valencia-Jaime, S. Singh, G. Avendaño-Franco, D. Raabe, and A. H. Romero, J. Alloys Compd. 691, 15 (2017).

[59] O. Karpinsky, L. Shelimova, M. Kretova, and J.-P. Fleurial, J. Alloys Compd. 268, 112 (1998).

[60] B. Sa, N. Miao, J. Zhou, Z. Sun, and R. Ahuja, Phys. Chem. Chem. Phys. 12, 1585 (2010).

[61] B. Sa, Z. Sun, T. Kaewmaraya, J. Zhou, and R. Ahuja, Sci. Adv. Mater. 5, 1493 (2013).

[62] R. Shaltaf, E. Durgun, J.-Y. Raty, P. Ghosez, and X. Gonze, Phys. Rev. B 78, 205203 (2008).

[63] K. Lee, E. D. Murray, L. Kong, B. I. Lundqvist, and D. C. Langreth, Phys. Rev. B 82, 081101 (2010).

[64] E.-R. Sittner, K. S. Siegert, P. Jost, C. Schlockermann, F. R. L. Lange, and M. Wuttig, Phys. Status Solidi A 210, 147 (2013).

[65] S. Singh, W. Ibarra-Hernández, I. Valencia-Jaime, G. AvendañoFranco, and A. H. Romero, Phys. Chem. Chem. Phys. 18, 29771 (2016). 